\title{
Fast neutrino flavor conversion: Roles of dense matter and spectrum crossing
}

\author{
Sajad Abbar ${ }^{*}$ and Huaiyu Duan ${ }^{\dagger}$ \\ Department of Physics \& Astronomy, University of New Mexico, Albuquerque, New Mexico 87131, USA
}

(Received 21 May 2018; published 16 August 2018)

\begin{abstract}
The flavor conversion of a neutrino usually occurs at densities $\lesssim G_{\mathrm{F}}^{-1} \omega$, whether in ordinary matter or a dense neutrino medium, and on time/distance scales of order $\omega^{-1}$, where $G_{\mathrm{F}}$ is the Fermi weak coupling constant and $\omega$ is the typical vacuum oscillation frequency of the neutrino. In contrast, fast neutrino flavor conversions or fast neutrino oscillations can occur on scales much shorter than $\omega^{-1}$ in a very dense, anisotropic neutrino gas such as that in a core-collapse supernova or a binary neutron star merger. The origin of fast neutrino oscillations still seems elusive except that it is a mathematical solution to the equation of motion. It has been suggested that the fast oscillations in stationary neutrino gases require a crossing in the electron lepton number angular distribution of the neutrino and that they are suppressed at large matter densities as normal oscillations are. By inspecting a simple four-beam neutrino model we illustrate how the multi-angle effects that were once found to suppress collective neutrino oscillations now give rise to fast flavor conversions. As a result, a large matter density can induce fast oscillations in certain astrophysical scenarios such as at the early epoch of a core-collapse supernova. We also provide an explicit proof of the necessity of a crossed neutrino angular distribution for fast oscillations to occur in an outward flowing, axially symmetric neutrino flux such as in the multi-bulb supernova model. However, fast oscillations can occur without a crossed angular distribution when both inward and outward flowing neutrino fluxes are present in a stationary neutrino gas.
\end{abstract}

DOI: 10.1103/PhysRevD.98.043014

\section{INTRODUCTION}

Neutrinos are copiously produced in core-collapse supernovae and binary neutron star mergers and play important roles in these astrophysical events. Because neutrinos can experience flavor transformation or oscillations during propagation (see, e.g., Ref. [1] for a review), it is of great interest to know whether neutrino oscillations can have any significant impact on the chemical evolution or even the dynamics of these environments.

In vacuum the flavor conversion of a neutrino occurs on a length scale determined by its vacuum oscillation frequency $\omega=\delta m^{2} / 2 E$, where $\delta m^{2}$ and $E$ are the (effective) mass-squared difference and the energy of the neutrino, respectively. For a $10 \mathrm{MeV}$ neutrino and the atmospheric mass splitting the flavor conversion occurs on the scale of $1 \mathrm{~km}$. A new length scale $\lambda^{-1}=\left(\sqrt{2} G_{\mathrm{F}} n_{e}\right)^{-1}$ comes into

*abbar@apc.in2p3.fr; Permenant address: Astro-Particule et Cosmologie (APC), Universit Denis Diderot, France.

duan@unm.edu

Published by the American Physical Society under the terms of the Creative Commons Attribution 4.0 International license. Further distribution of this work must maintain attribution to the author(s) and the published article's title, journal citation, and DOI. Funded by SCOAP. the play when the neutrino propagates through a dense matter such as inside the sun, where $G_{\mathrm{F}}$ is the Fermi weak coupling constant, and $n_{e}$ is the net electron number density. However, the neutrino flavor conversion is suppressed in dense matter until $\lambda \lesssim \omega$ where the MikheyevSmirnov-Wolfenstein (MSW) mechanism can operate [2]. As a result, the length scale of the neutrino flavor conversion through the MSW mechanism is also of order $\omega^{-1}$.

As early as in 2005 Sawyer has envisioned that flavor conversions can occur on the scale of $\left(\sqrt{2} G_{\mathrm{F}} n_{\nu}\right)^{-1}$ inside a core-collapse supernova, where $n_{\nu}$ is the number density of the neutrinos $[3,4]$. It is expected that this fast flavor conversion or fast (neutrino) oscillations can take place over the distance of $0.1-1 \mathrm{~cm}$. However, such fast oscillations were not observed in more sophisticated numerical simulations (see, e.g., Refs. [5-10]; see also Ref. [11] for a review). It turns out that all these calculations except those in Refs. [9,10] adopted the neutrino bulb model where the neutrinos of different flavors are emitted isotropically from the same neutrino sphere. Recently Sawyer reported fast flavor conversions in a multi-bulb model where different neutrino flavors have distinct neutrino spheres [12]. This finding seems to have been confirmed by other groups $[13,14]$.

Although a few works have been put out with the aims to understand fast neutrino flavor conversions from the 
perspective of the dispersion relation $[15,16]$ and in the nonlinear regime [17], the origin of fast oscillations remains largely elusive. In investigating a multibulb model with uniform neutrino angular distributions (on the surface of the neutrino spheres), Dasgupta et al. observed that "a necessary condition to have fast instabilities appear to be a crossing between the angular spectra of $\nu_{e}$ and $\bar{\nu}_{e}$ " [14]. However, this finding was not conclusive because no closed-form analytical solution was found. They have also concluded that "background matter suppresses instabilities in space (but not in time)". The main goals of this paper are to understand the origin of fast oscillations and to reexamine the above-mentioned two conclusions about fast oscillations of Ref. [14].

The rest of the paper is organized as follows. After establishing the formalism in Sec. II, we will revisit the bipolar model in Sec. III from the perspective of spectrum crossing. In Sec. IV we will explain the origin of fast oscillations in a four-beam neutrino model through its connection to the bipolar model. We will also give an explicit proof of the necessity of a crossed neutrino angular distribution for fast oscillations to occur in an outward flowing, axially symmetric neutrino flux. In Sec. V we will reexamine several important results about fast neutrino flavor conversions in the literature that are relevant to supernova physics. Finally, we will discuss the implications of our work and conclude in Sec. VI.

\section{EQUATION OF MOTION}

We consider the mixing between two neutrinos flavors, $e$ and $x$, where $x$ stands for an appropriate linear combination of the $\mu$ and $\tau$ flavors. We adopt the neutrino-flavor-isospin convention [18] in which the neutrino and antineutrino of energy $E$ are labeled by their oscillation frequencies

$$
\omega= \pm \frac{\delta m^{2}}{2 E},
$$

respectively, where the plus (minus) sign applies to the neutrino (antineutrino), and a positive (negative) neutrino mass-squared difference $\delta m^{2}$ indicates the normal (inverted) neutrino mass hierarchy.

We assume that the physical conditions including the densities of all neutrino species are essentially constant and homogeneous on the time and distance scales of interest. Following Refs. [15,19] we define the electron lepton number (ELN) distribution or spectrum as

$g(\omega, \mathbf{v}) \propto E^{2}\left|\frac{\mathrm{d} E}{\mathrm{~d} \omega}\right| \times \begin{cases}f_{\nu_{e}}(\mathbf{p})-f_{\nu_{x}}(\mathbf{p}) & \text { for neutrino, } \\ f_{\bar{\nu}_{x}}(\mathbf{p})-f_{\bar{\nu}_{e}}(\mathbf{p}) & \text { for antineutrino }\end{cases}$

where $\mathbf{v}$ and $\mathbf{p}$ are the velocity and momentum of the neutrino, respectively, and $f_{\nu_{\alpha} / \bar{\nu}_{\alpha}}(\alpha=e, x)$ are the occupation numbers of the corresponding neutrino species. Here we have assumed that neutrinos are relativistic so that $|\mathbf{v}|=1$ and $|\mathbf{p}|=E$. There exist multiple conventions in the literature for the normalization of the ELN spectrum. Here we choose to normalize it by the $\nu_{e}$ density such that

$$
\frac{n_{\nu_{e}}-n_{\nu_{x}}}{n_{\nu_{e}}}= \begin{cases}\int \frac{\mathrm{d} \mathbf{v}}{4 \pi} \int_{0}^{\infty} \mathrm{d} \omega g(\omega, \mathbf{v}) & \text { for } \mathrm{NH} \\ \int \frac{\mathrm{d} \mathbf{v}}{4 \pi} \int_{-\infty}^{0} \mathrm{~d} \omega g(\omega, \mathbf{v}) & \text { for } \mathrm{IH}\end{cases}
$$

where $n_{\nu_{e}}\left(n_{\nu_{x}}\right)$ is the total number density of $\nu_{e}\left(\nu_{x}\right)$, and $\mathrm{NH}$ and IH stand for the normal and inverted neutrino mass hierarchies, respectively. Note that, according to Eq. (1), the neutrino has a positive vacuum oscillation frequency $\omega$ if $\delta m^{2}>0$ and negative $\omega$ if $\delta m^{2}<0$. In the rest of the paper we will sometimes simply use the words "spectrum" and "distribution" to refer to the ELN energy or angular distributions.

We will examine the situation where neutrinos are almost in pure weak interaction states so that the (reduced, traceless) neutrino density matrix (in the weak interaction basis) is of the form

$$
\varrho_{\omega, \mathbf{v}} \approx\left[\begin{array}{cc}
1 & \epsilon_{\omega, \mathbf{v}} \\
\epsilon_{\omega, \mathbf{v}}^{*} & -1
\end{array}\right]
$$

where $|\epsilon| \ll 1$. We will look for the criteria for the onset of collective neutrino oscillations by performing the linear flavor-stability analysis in which the terms of order $\epsilon^{2}$ or higher are ignored [20].

In the absence of collisions neutrinos obey the equation of motion (EoM) [21]

$$
\left(\partial_{t}+\mathbf{v} \cdot \boldsymbol{\nabla}\right) \varrho_{\omega, \mathbf{v}}=-\mathrm{i}\left[\mathrm{H}_{0}+\mathrm{H}_{\nu \nu}, \varrho_{\omega, \mathbf{v}}\right],
$$

where $\mathrm{H}_{0}$ is the conventional neutrino oscillation Hamiltonian in the absence of ambient neutrinos, and $\mathrm{H}_{\nu \nu}$ is the potential due to neutrino-neutrino forward scattering. We assume a small neutrino mixing angle so that

$$
\mathrm{H}_{0} \approx(-\omega+\lambda) \frac{\sigma_{3}}{2},
$$

where $\lambda=\sqrt{2} G_{\mathrm{F}} n_{e}$ is (a measure of the strength of) the matter potential, and $\sigma_{3}=\operatorname{diag}[1,-1]$ is the third Pauli matrix. For the situation that we are interested in,

$$
\mathrm{H}_{\nu \nu}=\frac{\mu}{2} \int \frac{\mathrm{d} \mathbf{v}^{\prime}}{4 \pi} \int_{-\infty}^{\infty} \mathrm{d} \omega^{\prime}\left(1-\mathbf{v} \cdot \mathbf{v}^{\prime}\right) g^{\prime} \varrho^{\prime},
$$

where $\mu=\sqrt{2} G_{\mathrm{F}} n_{\nu_{e}}$ is (a measure of the strength of) the neutrino potential. For simplicity we sometimes suppress the arguments or subscripts of certain physical quantities, and the primed symbols such as $g^{\prime}=g\left(\omega^{\prime}, \mathbf{v}^{\prime}\right)$ 
are understood as the corresponding quantities that depend on the integration variables $\omega^{\prime}$ and $\mathbf{v}^{\prime}$. As usual we have ignored the trace terms which do not affect neutrino oscillations.

\section{FLAVOR INSTABILITIES IN HOMOGENEOUS AND ISOTROPIC NEUTRINO GASES}

It will prove helpful to first consider the relation between spectrum crossing and the flavor instabilities in a homogeneous and isotropic neutrino gas. The importance of spectrum crossing was first pointed out by Dasgupta et al. in a report of multiple spectral splits resulted from collective neutrino oscillations [19]. An explicit proof of this relation was later given by Banerjee et al. in an analysis on the flavor instabilities of dense neutrino gases [20]. As a useful example we will inspect the flavor instabilities in the bipolar model from the perspective of spectrum crossing. For completeness we will also include a brief proof of this relation for a neutrino gas with a continuous energy spectrum as that in Ref. [20] which will be used later in the paper.

\section{A. Bipolar model}

For a homogeneous and isotropic neutrino medium one has

$$
\varrho_{\omega, \mathbf{v}}(t, \mathbf{x})=\varrho_{\omega}(t), \quad g(\omega, \mathbf{v})=g(\omega)
$$

and

$$
\mathrm{i} \dot{\varrho}=\left[(-\omega+\lambda) \frac{\sigma_{3}}{2}+\frac{\mu}{2} \int_{-\infty}^{\infty} g^{\prime} \varrho^{\prime} \mathrm{d} \omega^{\prime}, \varrho\right] .
$$

The bipolar model describes a homogeneous and isotropic neutrino gas initially consisting of monoenergetic $\nu_{e}$ and $\bar{\nu}_{e}$ (see, e.g., Refs [22-24]). It has a discrete energy spectrum

$$
g(\omega)=\sum_{i=1,2} g_{i} \delta\left(\omega-\omega_{i}\right)
$$

where $g_{1}=1$ and $\omega_{1}=\delta m^{2} / 2 E_{1}$ for the neutrino with energy $E_{1}$, and $g_{2}=-n_{\bar{\nu}_{e}} / n_{\nu_{e}}$ and $\omega_{2}=-\omega_{1}$ for the antineutrino. Keeping only the linear terms in the EoM one has

$\mathrm{i}\left[\begin{array}{l}\dot{\epsilon}_{1} \\ \dot{\epsilon}_{2}\end{array}\right]=\left[\begin{array}{cc}-\omega_{1}+\lambda+\mu g_{2} & -\mu g_{2} \\ -\mu g_{1} & -\omega_{2}+\lambda+\mu g_{1}\end{array}\right]\left[\begin{array}{l}\epsilon_{1} \\ \epsilon_{2}\end{array}\right]$.

In the linear regime collective neutrino oscillations are represented by the normal modes

$$
\left[\begin{array}{l}
\epsilon_{1}(t) \\
\epsilon_{2}(t)
\end{array}\right]=\left[\begin{array}{l}
Q_{1} \\
Q_{2}
\end{array}\right] \exp (+\mathrm{i} \Omega t),
$$

where $Q_{i}(i=1,2)$ are two complex constants, and $\Omega$ is the collective oscillation frequency which can be solved from the characteristic equation

$$
\left(\Omega-\omega_{1}+\lambda+\mu g_{2}\right)\left(\Omega-\omega_{2}+\lambda+\mu g_{1}\right)-\mu^{2} g_{1} g_{2}=0 .
$$

The bipolar model becomes flavor unstable if there exists a solution with

$$
\kappa=-\operatorname{Im}(\Omega)>0 .
$$

From Eq. (13) it is straightforward to show that a flavor instability can exist only if

$$
\left[\left(\omega_{1}-\omega_{2}\right)+\left(g_{1}-g_{2}\right) \mu\right]^{2}<-4 g_{1} g_{2} \mu^{2},
$$

or, equivalently,

$\left(g_{1}+g_{2}\right)^{2} \mu^{2}+\left(\omega_{1}-\omega_{2}\right)^{2}<-2\left(\omega_{1}-\omega_{2}\right)\left(g_{1}-g_{2}\right) \mu$.

A few remarks are in order.

(i) Because inequalities (15) do not depend on $\lambda$, the presence of a uniform matter density does not affect flavor instability. In fact, it is obvious from Eq. (11) that the presence of the ambient matter only shifts the collective oscillation frequency $\Omega$ by $-\lambda$ [18].

(ii) Eq. (11) implies that, if the oscillation frequencies of both neutrino modes are shifted by a common value $\Delta \Omega$, then the collective oscillation frequency $\Omega$ is also shifted by $\Delta \Omega$ which again does not affect the flavor instability [18].

(iii) Eq. (15a) implies that a neutrino flavor instability exists only if the ELNs of the two neutrino modes, $g_{1}$ and $g_{2}$, have opposite signs, i.e., $g_{1} g_{2}<0$. (See Fig. 1.)

(iv) Eq. (15b) gives another necessary condition of flavor instability, $\left(\omega_{1}-\omega_{2}\right)\left(g_{1}-g_{2}\right)<0$. For the bipolar system that we considers, this is possible only if $\omega_{1}<0$ which requires $\delta m^{2}<0$, i.e., the neutrino mass hierarchy is inverted. ${ }^{1}$

If the neutrino has the inverted mass hierarchy, it is straightforward to show from Eq. (15) that the bipolar model is unstable for $\mu \in\left(\mu_{-}, \mu_{+}\right)$, where the lower and upper boundaries of the instability region are

\footnotetext{
${ }^{1}$ In Ref. [19] and some other references, $\omega$ is defined to be always positive for the neutrino. In that convention, an energy spectrum with a negative (positive) crossing can be unstable if the neutrino has a normal (inverted) neutrino mass hierarchy. In our convention, however, $g(\omega) \rightarrow g(-\omega)$ as the neutrino mass hierarchy is flipped, and a negative crossing in the energy spectrum becomes positive and vice versa. The flavor stabilities will change accordingly. Either way, a neutrino system with a single crossed energy spectrum can be unstable with one neutrino mass hierarchy but stable with the other.
} 
postive crossing

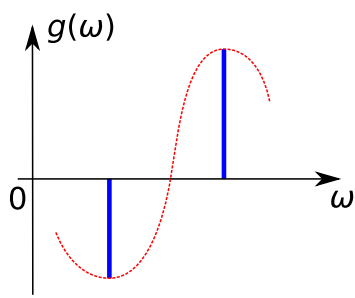

negative crossing

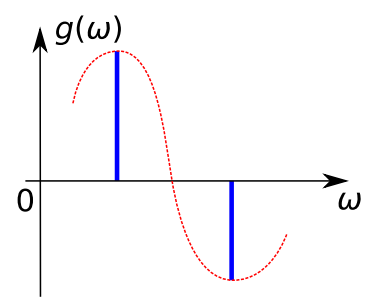

FIG. 1. Illustrative discrete (thick solid lines) and continuous (dashed curves) electron lepton number (ELN) spectra $g(\omega)$ with positive and negative crossings, respectively, where $\omega$ is the oscillation frequency of the neutrino. For the discrete ELN spectrum, the lengths of the thick solid lines represent the coefficients in front of the delta functions in Eq. (10). Collective oscillations can occur in a dense neutrino medium only if its initial ELN spectrum possesses one or multiple negative crossings.

$$
\mu_{ \pm}=\frac{\left|\omega_{1}-\omega_{2}\right|}{\left(\sqrt{\left|g_{1}\right|} \mp \sqrt{\left|g_{2}\right|}\right)^{2}},
$$

respectively. Unless $\left|g_{1}\right|$ and $\left|g_{2}\right|$ are almost the same, $\mu_{+}$ and $\mu_{-}$are of the same magnitude as the spread of the oscillation frequencies $\Delta \omega=\left|\omega_{1}-\omega_{2}\right|$. By the dimension argument one expects the maximum value of $\kappa$ to be of the same magnitude as $\Delta \omega$, which in turn is usually of the same order as $\left|\omega_{1}\right|$. Therefore, like the MSW mechanism, the flavor conversion in the bipolar model, and also in homogeneous and isotropic neutrino media in general, usually occurs at neutrino densities $\lesssim G_{\mathrm{F}}^{-1} \omega$ and on time/ distance scales of order $\omega^{-1}$ with $\omega$ being a characteristic vacuum oscillation frequency of the neutrino gas.

\section{B. Neutrino gas of a continuous spectrum ${ }^{2}$}

The linearized EoM of a homogeneous and isotropic neutrino gas with a continuous energy spectrum is

$$
\mathrm{i} \dot{\epsilon}=(-\omega+\bar{\lambda}) \epsilon-\mu \int_{-\infty}^{\infty} g^{\prime} \epsilon^{\prime} \mathrm{d} \omega^{\prime}
$$

where $\bar{\lambda}=\lambda+\Phi_{0}$ with

$\Phi_{0}=\mu \int_{-\infty}^{\infty} g(\omega) \mathrm{d} \omega=\sqrt{2} G_{\mathrm{F}}\left[\left(n_{\nu_{e}}-n_{\nu_{x}}\right)-\left(n_{\bar{\nu}_{e}}-n_{\bar{\nu}_{x}}\right)\right]$.

Assuming solution $\epsilon_{\omega}(t)=Q_{\omega} e^{i \Omega t}$ to Eq. (17) one obtains

\footnotetext{
${ }^{2}$ The proof for the necessity of a crossed spectrum for neutrino flavor instability was first given by Banerjee et al. in Ref. [20]. The content of this section is included for completeness.
}

$$
(\Omega+\bar{\lambda}-\omega) Q=\mu \int_{-\infty}^{\infty} g^{\prime} Q^{\prime} \mathrm{d} \omega^{\prime}
$$

which implies

$$
Q_{\omega} \propto \frac{1}{\Omega+\bar{\lambda}-\omega} .
$$

Again one sees that, $\bar{\lambda}$, a combined potential due to the matter and neutrino background, has the effect of shifting the collective oscillation frequency $\Omega$ but does not affect the flavor instability. For the purpose of analyzing flavor instabilities we will assume $\bar{\lambda}=0$ in this section. Substituting Eq. (20) back into Eq. (19) one obtains

$$
\int_{-\infty}^{\infty} \frac{g(\omega) \mathrm{d} \omega}{(\omega-\gamma)+\mathrm{i} \kappa}=-\frac{1}{\mu}
$$

where $\gamma=\operatorname{Re}(\Omega)$.

Suppose that there exists a flavor instability for $\mu$ within the range of $\left(\mu_{-}, \mu_{+}\right)$. In the limit $\mu \rightarrow \mu_{-}+0^{+}, \kappa \rightarrow 0^{+}$, and Eq. (21) becomes

$$
\mathcal{P} \int_{-\infty}^{\infty} \frac{g(\omega) \mathrm{d} \omega}{\omega-\omega_{\mathrm{c}}}-\mathrm{i} \pi g\left(\omega_{\mathrm{c}}\right)=-\frac{1}{\mu_{-}},
$$

where $\mathcal{P}$ signifies the Cauchy principal value of the integral, and $\omega_{\mathrm{c}}=\left.\Omega\right|_{\mu=\mu_{-}} \cdot{ }^{3}$ Because $\mu$ is real, Eq. (22) implies that the spectrum $g(\omega)$ must cross 0 at $\omega=\omega_{\mathrm{c}}$. In addition, unless there are other crossing points nearby, the integral in Eq. (22) has the same sign as the crossing of $g(\omega)$ at $\omega_{\mathrm{c}}$. This in turn implies that the spectrum crossing at $\omega_{\mathrm{c}}$ must be negative because we have defined $\mu$ to be positive. This result can be considered as a generalization of that in the bipolar model. (See Fig. 1.)

\section{FAST FLAVOR CONVERSIONS IN ANISOTROPIC NEUTRINO MEDIA}

We will now turn to the relation between fast flavor conversions and the crossings in the neutrino angular distribution. The first, albeit inconclusive, observation of this relation was made by Dasgupta et al. in a study of fast neutrino oscillations in a multibulb supernova model [14]. Here we will consider stationary, anisotropic neutrino media which consist of neutrino fluxes emitted constantly from planar surfaces. We assume that the neutrino emission is uniform on the emission plane and that the matter distribution is homogeneous in the whole space. We will

\footnotetext{
${ }^{3}$ Here we have assumed that, if $g(\omega)$ is nonzero only within range $\left(\omega_{\min }, \omega_{\max }\right)$, then $\omega_{\min }<\omega_{\mathrm{c}}<\omega_{\max }$. This is a reasonable assumption because $\mu_{-}$is small and, therefore, collective oscillation frequency $\Omega$ at $\mu=\mu_{-}$is an "average" value of the oscillation frequency $\omega$ which should be within the range of $\left(\omega_{\min }, \omega_{\max }\right)$.
} 


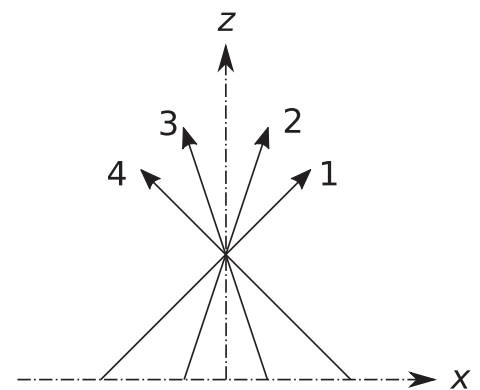

FIG. 2. A schematic diagram of the four-beam model in which the neutrinos of four momentum modes are emitted constantly and homogeneously from the $x-y$ plane in the directions that are parallel to the $x-z$ plane.

first use the four-beam neutrino model to demonstrate the origin of fast flavor conversions by relating it to the bipolar model. We will then give an explicit proof of the necessity of a crossed neutrino angular distribution for fast oscillations to occur in an outward flowing, axially symmetric neutrino flux.

\section{A. Four-beam model}

The four-beam neutrino model has the ELN distribution

$$
g(\mathbf{v}, \omega)=4 \pi \sum_{i=1}^{4} g_{i} \delta\left(\mathbf{v}-\mathbf{v}_{i}\right) \delta\left(\omega-\omega_{i}\right)
$$

where $\mathbf{v}_{i}$ and $\omega_{i}(i=1,2,3,4)$ are the velocities and oscillation frequencies of the four neutrino modes of the system. (See Fig. 2). We will assume the reflection symmetry between neutrino modes $(1,2)$ and $(4,3)$ in the four-beam model except for small differences among $\varrho_{i}$, i.e., $\left(\epsilon_{1}, \epsilon_{2}\right) \neq\left(\epsilon_{4}, \epsilon_{3}\right)$. We define

$\epsilon_{1 \pm}(z)=\frac{\epsilon_{1}(z) \pm \epsilon_{4}(z)}{2}, \quad \epsilon_{2 \pm}(z)=\frac{\epsilon_{2}(z) \pm \epsilon_{3}(z)}{2}$,

where the $z$ direction is perpendicular to the neutrino emission plane. Because of the reflection symmetry in the EoM, the symmetric modes $\left(\epsilon_{i+}\right)$ and the antisymmetric modes $\left(\epsilon_{i-}\right)$ evolve independently in the linear regime.

\section{Symmetric mode} EoM

$\mathrm{i} \frac{\mathrm{d}}{\mathrm{d} z}\left[\begin{array}{c}\epsilon_{1+} \\ \epsilon_{2+}\end{array}\right]=\left[\begin{array}{cc}-\tilde{\omega}_{1+}+\tilde{\mu}_{+} \tilde{g}_{2+} & -\tilde{\mu}_{+} \tilde{g}_{2+} \\ -\tilde{\mu}_{+} \tilde{g}_{1+} & -\tilde{\omega}_{2+}+\tilde{\mu}_{+} \tilde{g}_{1+}\end{array}\right]\left[\begin{array}{c}\epsilon_{1+} \\ \epsilon_{2+}\end{array}\right]$

in the linear regime, where

$$
\begin{array}{ll}
\tilde{\omega}_{1+}=\frac{\omega_{1}-\lambda}{v_{1 z}}, & \tilde{g}_{1+}=\frac{g_{1}}{v_{2 z}}, \\
\tilde{\omega}_{2+}=\frac{\omega_{2}-\lambda}{v_{2 z}}, & \tilde{g}_{2+}=\frac{g_{2}}{v_{1 z}}, \\
\tilde{\mu}_{+}=\mu_{12}+\mu_{13} &
\end{array}
$$

with $v_{z}$ being the $z$ component of $\mathbf{v}$, and $\mu_{i j}=$ $\left(1-\mathbf{v}_{i} \cdot \mathbf{v}_{j}\right) \mu$. Here we have rewritten the EoM of the symmetric mode in a form similar to that of the bipolar model, Eq. (11), so that the results of the bipolar model can be applied here. Equation (26) shows that the presence of the ordinary matter can contribute to the spread of effective oscillation frequencies $\Delta \tilde{\omega}=\left|\tilde{\omega}_{1+}-\tilde{\omega}_{2+}\right|$, which is generally true for a system with an anisotropic neutrino emission. This multiangle matter effect is responsible for the suppression of neutrino oscillations in the accretion phase of a core-collapse supernova if all neutrino species are emitted from the same neutrino sphere and with the same angular distribution [25].

Here we want to emphasize that the same multiangle matter effect can also induce fast flavor conversions. To see this we take the limit $\delta m^{2} \rightarrow 0$ so that

$$
\tilde{\omega}_{1+} \rightarrow-\frac{\lambda}{v_{1 z}}, \quad \tilde{\omega}_{2+} \rightarrow-\frac{\lambda}{v_{2 z}} .
$$

The discussion in Sec. III A implies that there can exist flavor instability even in this limit. Assuming $v_{1 z}<v_{2 z}$, the requirement of a negatively crossed effective spectrum $\tilde{g}_{+}\left(\tilde{\omega}_{+}\right)$means that $g_{1}>0>g_{2}$, e.g., beams $(1,4)$ are emitted in the $\nu_{e}$ state and beams $(2,3)$ in the $\bar{\nu}_{e}$ state. In other words, the existence of a flavor instability in the symmetric mode requires that there exist a negative crossing in the angular distribution

$$
G\left(v_{z}\right)=\int_{-\infty}^{\infty} g\left(v_{z}, \omega\right) \mathrm{d} \omega .
$$

When this is the case the neutrino medium becomes unstable against the flavor conversion when $\mu$ is comparable to $\lambda$. [See Eqs. (16) and (27).] The exponential growth rate $\kappa$ of the flavor instability is also of the same order of $\lambda$ which can be much larger than vacuum oscillation frequencies (taken to be zero in this limit).

As an example we consider the case where beams $(1,4)$ are emitted in the $\nu_{e}$ state with $v_{1 z}=1 / 2$ and $g_{1}=1$ and beams $(2,3)$ in the $\bar{\nu}_{e}$ state with various values of $v_{2 z}$ and $g_{2}$. In the two top panels of Fig. 3 we plot the exponential growth rate $\kappa$ of the flavor instability as a function of $v_{2 z}$ and $g_{2}$ with fixed ratios $\lambda / \mu=1$ and 3 , respectively. We only plotted the results with $g_{2}<0$ where there exits a crossing in the effective spectrum $\tilde{g}_{+}\left(\tilde{\omega}_{+}\right)$. From the figure one sees that the symmetric fast flavor conversions are 

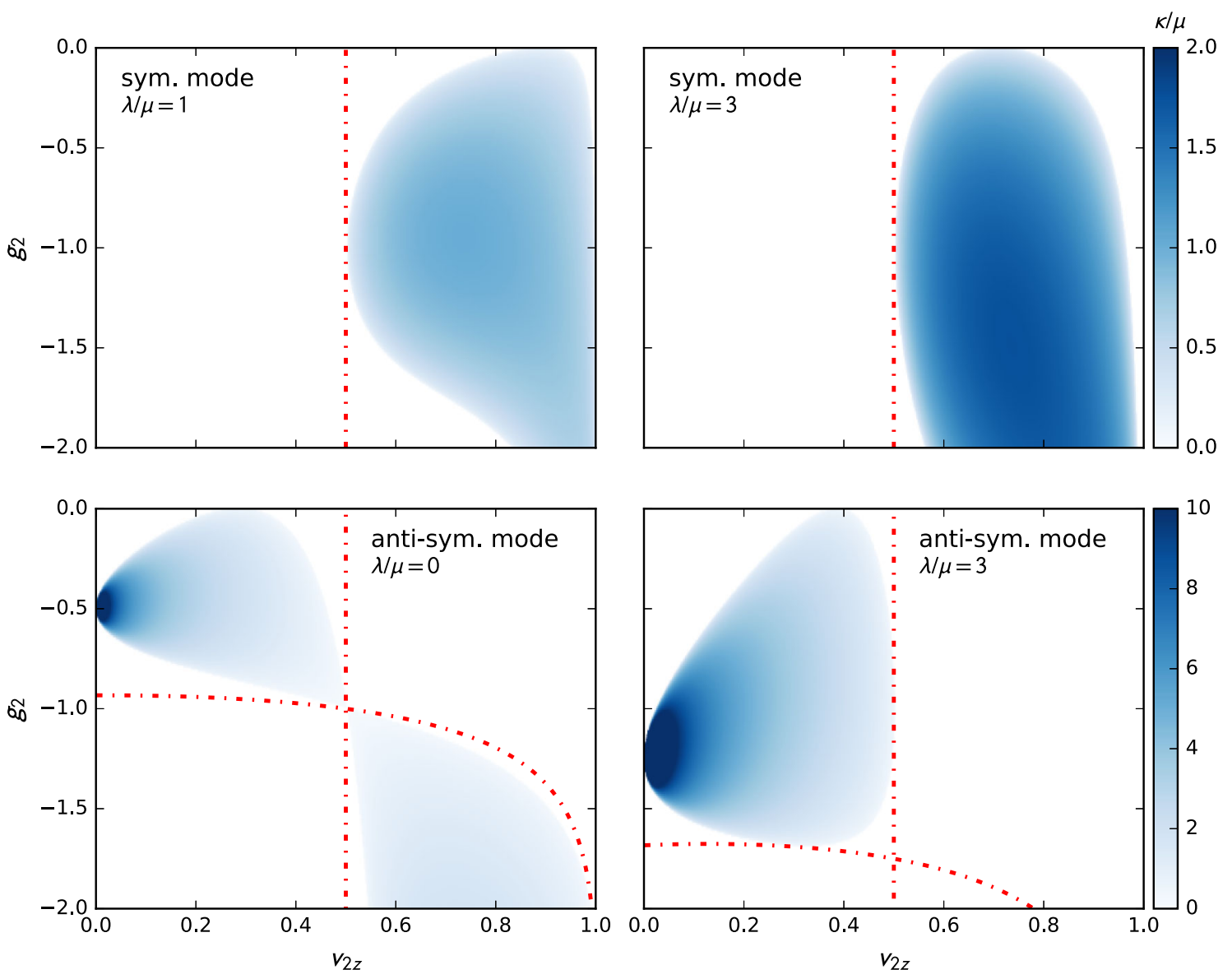

FIG. 3. Flavor instabilities in the four-beam neutrino model in Fig. 2 for the neutrino oscillation modes which preserve the reflection symmetry (top panels) and break this symmetry (bottom panels), respectively. The color density indicates the exponential growth rate $\kappa$ of the flavor instability of the neutrino medium as a function of the electron lepton numbers (ELNs) $g_{2}=g_{3}$ and the $z$-component velocities $v_{2 z}=v_{3 z}$ of two of the neutrino modes. The other two modes have fixed values of $g_{1}=g_{4}=1$ and $v_{1 z}=v_{4 z}=1 / 2$. The dotdashed curves mark the boundaries between the scenarios where the effective ELN spectra have negative and positive crossings, respectively. Both $\kappa$ and the strength of the matter potential $\lambda$ (as indicated) are measured in the strength of the neutrino potential $\mu$.

possible in the four-beam model only if the $\bar{\nu}_{e}$ beams are emitted in a more forward direction than the $\nu_{e}$ beams so that $\tilde{g}_{+}\left(\tilde{\omega}_{+}\right)$has a negative crossing.

\section{Antisymmetric mode}

The EoM for the antisymmetric mode is the same as Eq. (25) except that the "plus" quantities are replaced by their "minus" counterparts:

$$
\begin{array}{ll}
\tilde{\omega}_{1-}=\frac{\omega_{1}-\lambda-2 \mu_{14} g_{1}-2 \mu_{13} g_{2}}{v_{1 z}}, & \tilde{g}_{1-}=-\frac{g_{1}}{v_{2 z}}, \\
\tilde{\omega}_{2-}=\frac{\omega_{2}-\lambda-2 \mu_{13} g_{1}-2 \mu_{23} g_{2}}{v_{2 z}}, & \tilde{g}_{2-}=-\frac{g_{2}}{v_{1 z}}, \\
\tilde{\mu}_{-}=-\mu_{12}+\mu_{13} . &
\end{array}
$$

Without losing generality we will assume the configuration with $\mathbf{v}_{1} \cdot \mathbf{v}_{2}>\mathbf{v}_{1} \cdot \mathbf{v}_{3}$ so that $\tilde{\mu}_{-}>0$. The comparison between Eq. (29) and Eq. (26) reveals two important features of the antisymmetric collective mode in the fourbeam model:

(i) Both the ordinary matter and the background neutrino medium contribute to the spread of effective oscillation frequencies $\Delta \tilde{\omega}_{-}=\left|\tilde{\omega}_{1-}-\tilde{\omega}_{2-}\right|$. This multiangle effect due to the neutrino medium itself can suppress collective neutrino oscillations in the supernova model in which all neutrino species have the same angular distribution [8].

(ii) The effective spectrum $\tilde{g}_{-}\left(\tilde{\omega}_{-}\right)$has a sign opposite to that of the actual ELN distribution. This implies that a flavor instability may exist when there is a positive crossing in the angular distribution $G\left(v_{z}\right)$.

As in the case of the symmetric mode, there can exist a flavor instability for the antisymmetric mode in the limit $\delta m^{2} \rightarrow 0$. A crossing in the effective ELN spectrum $\tilde{g}_{-}\left(\tilde{\omega}_{-}\right)$ (i.e., $\left.\tilde{g}_{1-} \tilde{g}_{2_{-}}<0\right)$ also implies a crossing in the actual angular distribution $G\left(v_{z}\right)\left(g_{1} g_{2}<0\right)$. Unlike the symmetric mode, the antisymmetric mode can have fast flavor 
conversions even when $\lambda=0$. In addition, the multiangle neutrino effect can change the order of $\tilde{\omega}_{i-}$, i.e., whether $\tilde{\omega}_{1-}<\tilde{\omega}_{2-}$ or $\tilde{\omega}_{1-}>\tilde{\omega}_{2_{-}}$. As a result, the regimes where the effective spectrum $\tilde{g}_{-}\left(\tilde{\omega}_{-}\right)$has a negative crossing are not solely determined by $v_{2 z}$ as in the case of the symmetric mode. In the lower panels of Fig. 3 we plot the exponential growth rate $\kappa$ of this instability as a function of $v_{2 z}$ and $g_{2}$ with fixed ratios $\lambda / \mu=0$ and 3 , respectively.

\section{B. Outward flowing, axially symmetric neutrino flux}

We next consider an outward flowing, nearly axially symmetric neutrino flux with

$$
g(\mathbf{v}, \omega)=g\left(v_{z}, \omega\right)
$$

and small perturbations to the initial states of the neutrinos which can depend the azimuthal angle $\varphi$ of $\mathbf{v}$. We will again consider flavor instabilities in the limit $\delta m^{2} \rightarrow 0$. The linearized EoM of this model is

$\mathrm{i} v_{z} \frac{\mathrm{d} \epsilon}{\mathrm{d} z}=\left(\lambda+\Phi_{0}-v_{z} \Phi_{z}\right) \epsilon-\mu \int\left(1-\mathbf{v} \cdot \mathbf{v}^{\prime}\right) G^{\prime} \epsilon^{\prime} \frac{\mathrm{d} \mathbf{v}^{\prime}}{4 \pi}$,

where

$$
\Phi_{0}=\mu \int G^{\prime} \frac{\mathrm{d} \mathbf{v}^{\prime}}{4 \pi}, \quad \Phi_{z}=\mu \int v_{z}^{\prime} G^{\prime} \frac{\mathrm{d} \mathbf{v}^{\prime}}{4 \pi} .
$$

Following Ref. [20] we assume a solution of the form

$$
\epsilon_{\mathbf{v}}(z)=\frac{Q_{\mathbf{v}}}{v_{z}} e^{\mathrm{i} \Omega z}
$$

to Eq. (31) and obtain

$$
\left(\Omega-\Phi_{z}+\frac{\bar{\lambda}}{v_{z}}\right) Q=\mu \int\left(1-\mathbf{v} \cdot \mathbf{v}^{\prime}\right) G^{\prime} \frac{Q^{\prime}}{v_{z}^{\prime}} \frac{\mathrm{d}^{\prime}}{4 \pi},
$$

where $\bar{\lambda}=\lambda+\Phi_{0}$. Comparing Eqs. (19) and (34) one sees that $\Phi_{z}$ shifts the collective oscillation frequency $\Omega$ while $-\bar{\lambda} / v_{z}$ plays the role of vacuum oscillation frequency $\omega$. Equation (34) implies

$$
Q_{\mathbf{v}}=\frac{A+B v_{z}+C \sqrt{u} \cos \varphi+D \sqrt{u} \sin \varphi}{\Omega-\Phi_{z}+\bar{\lambda} / v_{z}},
$$

where

$$
\begin{aligned}
& A=\mu \int G^{\prime} \frac{Q^{\prime}}{v_{z}^{\prime}} \frac{\mathrm{d} \mathbf{v}^{\prime}}{4 \pi}, \\
& B=-\mu \int G^{\prime} Q^{\prime} \frac{\mathrm{d} \mathbf{v}^{\prime}}{4 \pi},
\end{aligned}
$$

$$
\begin{aligned}
& C=-\mu \int \sqrt{u^{\prime}} \cos \varphi^{\prime} G^{\prime} \frac{Q^{\prime}}{v_{z}^{\prime}} \frac{\mathrm{d} \mathbf{v}^{\prime}}{4 \pi}, \\
& D=-\mu \int \sqrt{u^{\prime}} \sin \varphi^{\prime} G^{\prime} \frac{Q^{\prime}}{v_{z}^{\prime}} \frac{\mathrm{d} \mathbf{v}^{\prime}}{4 \pi},
\end{aligned}
$$

and $u=1-v_{z}^{2}$. Substituting Eq. (35) to Eq. (36) we obtain

$$
\left[\begin{array}{cccc}
I[1]-1 & I\left[v_{z}\right] & 0 & 0 \\
-I\left[v_{z}\right] & -I\left[v_{z}^{2}\right]-1 & 0 & 0 \\
0 & 0 & -\frac{1}{2} I[u]-1 & 0 \\
0 & 0 & 0 & -\frac{1}{2} I[u]-1
\end{array}\right]\left[\begin{array}{c}
A \\
B \\
C \\
D
\end{array}\right]=0
$$

where

$$
I\left[f\left(v_{z}\right)\right]=\frac{\mu}{2} \int_{0}^{1} \frac{G\left(v_{z}\right) f\left(v_{z}\right)}{\Omega-\Phi_{z}+\bar{\lambda} / v_{z}} \frac{\mathrm{d} v_{z}}{v_{z}} .
$$

Equation (37) has a solution only if

$$
(I[1]-1)\left(I\left[v_{z}^{2}\right]+1\right)=I^{2}\left[v_{z}\right]
$$

or

$$
I[u]=-2,
$$

which corresponds to the collective modes that preserve and break the axial symmetry, respectively. ${ }^{4}$

Let us consider the symmetry-breaking solution first. We rewrite Eq. (39b) as

$$
\int_{\tilde{\omega}_{\min }}^{\tilde{\omega}_{\max }} \frac{\tilde{g}(\tilde{\omega}) \mathrm{d} \tilde{\omega}}{\left[\tilde{\omega}-\left(\gamma-\Phi_{z}\right)\right]+\mathrm{i} \kappa}=\frac{1}{\mu},
$$

where

$$
\tilde{\omega}=-\frac{\bar{\lambda}}{v_{z}}, \quad \tilde{g}(\tilde{\omega})=v_{z}\left(1-v_{z}^{2}\right) \frac{G\left(v_{z}\right)}{4 \bar{\lambda}},
$$

and $\left(\tilde{\omega}_{\min }, \tilde{\omega}_{\max }\right)=(-\bar{\lambda}, \infty)$ if $\bar{\lambda}<0$ and $(-\infty,-\bar{\lambda})$ if $\bar{\lambda}>0$. Using the result of the homogeneous, isotropic medium (in Sec. III B), we determine that fast flavor conversions that break the axial symmetry can occur in this medium only if there exists a positive crossing in the angular distribution $G\left(v_{z}\right)$ within $[0,1]$.

One can also perform a similar analysis to Eq. (39a) and show that fast flavor conversions that preserve the axial symmetry can take place if there exists a crossing in $G\left(v_{z}\right)$,

\footnotetext{
${ }^{4}$ The derivation of the characteristic equation (39) for this model is similar to that in Refs. $[13,14]$ and is included here for completeness.
} 
although the sign of the crossing is not constrained. (See the Appendix for details.)

\section{MULTIBULB SUPERNOVA MODEL}

Now we consider the simple supernova model proposed by Sawyer in Ref. [12] in which $\nu_{e}, \bar{\nu}_{e}, \nu_{x}$ are emitted constantly and half-isotropically from three spherically symmetric neutrino spheres or "bulbs" of radii $R_{\nu_{e}}, R_{\bar{\nu}_{e}}$ and $R_{\nu_{x}}$, respectively, and $\bar{\nu}_{x}$ has the identical emission characteristics as $\nu_{x}$. This multibulb model is a generalization of the neutrino (single-)bulb model in Ref. [5] where $R_{\nu_{e}}=R_{\bar{\nu}_{e}}=R_{\nu_{x}}$. Because the typical length scale of the fast flavor conversion is much smaller than $R_{\nu}\left(\nu=\nu_{e}, \bar{\nu}_{e}\right.$, $\nu_{x}$ ), we can ignore the spherical nature of the model and apply the results of Sec. IV B.

In the multibulb model the number flux of the neutrino species at radius $r$ takes the form

$$
j_{\nu}(r, \vartheta)=\frac{n_{\nu, 0}}{2 \pi} \Theta\left(R_{\nu}-r \sin \vartheta\right),
$$

where $\vartheta$ is the polar angle of the flux with respect to the radial direction, and $n_{\nu, 0}$ is the number density of the neutrino species $\nu$ on the corresponding neutrino sphere. The total number luminosity of neutrino species $\nu$ is

$$
\dot{N}_{\nu}=4 \pi r^{2} \int_{0}^{2 \pi} \mathrm{d} \varphi \int_{0}^{1} \mathrm{~d}(\cos \vartheta) j_{\nu}(r, \vartheta) \cos \vartheta=2 \pi R^{2} n_{\nu, 0} .
$$

For $R_{\nu_{e}}>R_{\bar{\nu}_{e}}>R_{\nu_{x}}$ and $\dot{N}_{\nu_{x}}: \dot{N}_{\bar{\nu}_{e}}: \dot{N}_{\nu_{e}}=0.62: 0.77: 1$ Sawyer found that there could exist a flavor instability even on the $\nu_{e}$ sphere if

$$
\frac{R_{\bar{\nu}_{e}}}{R_{\nu_{e}}}>0.44+0.55\left(\frac{R_{\nu_{x}}}{R_{\nu_{e}}}\right) .
$$

For $\quad R_{\nu_{e}}=R_{\bar{\nu}_{e}} / 0.93=R_{\nu_{x}} / 0.8=15 \mathrm{~km} \quad$ and $\quad n_{\bar{\nu}_{e}, 0}=$ $10^{32} \mathrm{~cm}^{-3}$ he calculated the instability growth rate to be $\kappa=0.32 \mathrm{~m}^{-1}$ on the surface of the $\nu_{e}$ sphere. However, since $j_{\nu_{x}}=j_{\bar{\nu}_{x}}$ in the multibulb model and, therefore, the contribution of $\nu_{x}$ and $\bar{\nu}_{x}$ to the angular distribution $G\left(v_{z}\right)$ cancel each other, the criterion for "pure" fast neutrino flavor conversions (with $\delta m^{2} \rightarrow 0$ ) should not depend on $R_{\nu_{x}}$ or $n_{\nu_{x}, 0}$, which is contrary to the condition set forth in Eq. (44). (For this reason the multibulb model was called the two-bulb model by Chakraborty et al. in Ref. [13].) Further, it was observed by Dasgupta et al. [14] that, in order for fast flavor conversions to take place in the multibulb model, there should exist at least one crossing in the angular distribution. This requirement has been explicitly shown in Sec. IV B, and implies that

$$
\left(n_{\nu_{e}, 0}-n_{\bar{\nu}_{e}, 0}\right)\left(R_{\nu_{e}}-R_{\bar{\nu}_{e}}\right)<0 .
$$

The example given above by Sawyer in Ref. [12] does not satisfy this criterion. He also did not include the matter effect which can significantly affect the fast neutrino flavor conversion. We have performed a linear flavor stability analysis to the multibulb model, and we did not find any flavor instability with the parameters given in Ref. [12].

In Ref. [13] Chakraborty et al. applied the linear flavor instability analysis to the multibulb model but only in the far regime where $r \gg R_{\nu}$. In this regime

$$
2 \pi j_{\nu}(r, \vartheta) \mathrm{d}(\cos \vartheta) \approx n_{\nu}(r, u)(-\mathrm{d} u),
$$

where $u=\left.\sin ^{2} \vartheta\right|_{r=R_{\star}}$, and $n_{\nu_{e}}$ and $n_{\bar{\nu}_{e}}$ are parametrized as

$$
n_{\nu} \propto \frac{1 \pm a}{1 \pm b} \times \begin{cases}1 & \text { for } 0<u<1 \pm b \\ 0 & \text { otherwise }\end{cases}
$$

with the upper and lower signs applying to $\nu_{e}$ and $\bar{\nu}_{e}$, respectively. In the above parametrization, the asymmetry parameter $a$ and the width parameter $b$ are both within the range of $(-1,1)$ and determine $\dot{N}_{\nu_{e}} / \dot{N}_{\bar{\nu}_{e}}$ and $R_{\nu_{e}} / R_{\bar{\nu}_{e}}$, respectively, and $R_{\star}$ is the radius at which the above parametrization is valid. In this parametrization, the angular distribution has a crossing only if

$$
(a-b) b<0 .
$$

Indeed, Figs. 5 and 6 of Ref. [13] show that the flavor instabilities exist only in the parameter space where the above criterion is satisfied. Those figures also demonstrate that a large matter density (i.e., $\lambda \gtrsim \mu$ ) can dramatically affect the neutrino flavor instabilities in the multibulb model.

As another example of the multi-bulb model, we calculated the neutrino flavor instability region in a onedimensional, electron-capture supernova model with an $8.8 M_{\odot}$ progenitor which was computed by the Garching group [26]. At $t \approx 200 \mathrm{~ms}$ after the core bounce this model has $\dot{N}_{\nu_{e}}=9.80 \times 10^{56} \mathrm{~s}^{-1}, \dot{N}_{\bar{\nu}_{e}}=8.36 \times 10^{56} \mathrm{~s}^{-1}, \dot{N}_{\nu_{x}}=$ $6.92 \times 10^{56} \mathrm{~s}^{-1}, \quad R_{\nu_{e}}=40.2 \mathrm{~km}, \quad R_{\bar{\nu}_{e}}=36.6 \mathrm{~km} \quad$ and $R_{\nu_{x}}=36.1 \mathrm{~km}$, respectively. It is easy to check that the neutrino gas in this supernova model has a crossed angular distribution. Using the results in Sec. IV B we plot the flavor unstable region in the supernova as the light shadowed area in Fig. 4 for various matter densities. To simplify the calculation, we have assumed the two-flavor mixing with the inverted neutrino mass hierarchy and a single vacuum oscillation frequency $\omega_{0}=0.68 \mathrm{~km}^{-1}$ for all neutrino species. This simplification should not have a dramatic impact on the flavor unstable region in Fig. 4 which is a log-log plot. We depict the flavor unstable region of the pure fast modes with $\delta m^{2} \rightarrow 0$ as the hatched area in the same figure. As comparison we also plot the flavor unstable region as dark shadowed region for the case where the neutrino spheres of all other neutrino species coincide 


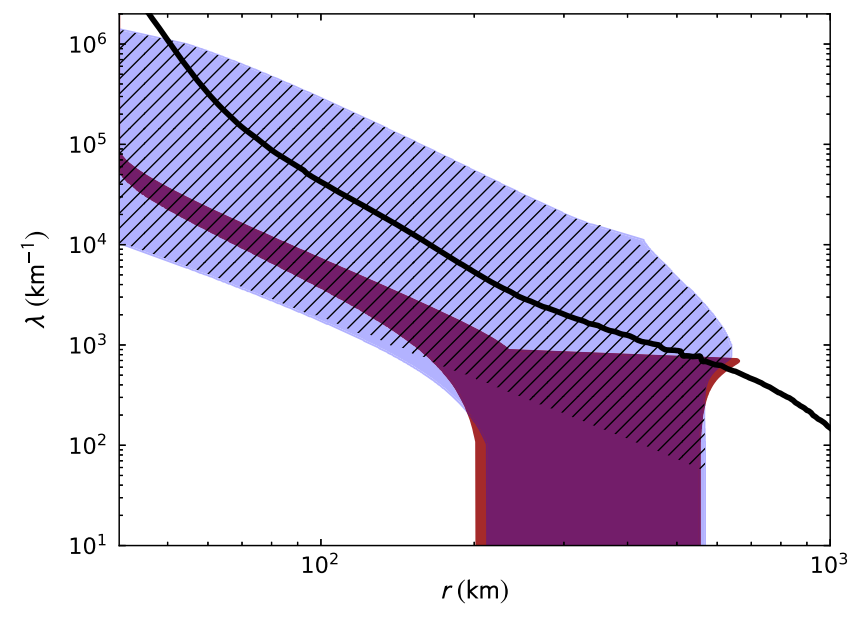

FIG. 4. The flavor instability region of the neutrino gas (in the light shadow) with various matter densities (proportional to the matter potential $\lambda$ ) in a one-dimensional, electron-capture supernova model at $t \approx 200 \mathrm{~ms}$ after the core bounce [26]. The thick solid curve is the actual matter profile as a function of radius $r$. The hatched region is where the "pure" fast flavor conversions (with the neutrino mass-squared splitting $\delta m^{2} \rightarrow 0$ ) become possible, and the dark shadowed region is where collective neutrino oscillations can occur if the neutrino spheres of $\nu_{x}$ and $\bar{\nu}_{e}$ coincide with that of $\nu_{e}$.

with that of $\nu_{e}$. This corresponds to the single-bulb model where only "slow" neutrino oscillations can exist since there is no crossing in the angular distribution. Finally we plot the actual matter profile as the thick solid curve in the figure.

Figure 4 clearly demonstrates the important influence of the ambient matter on neutrino oscillations in a supernova. In the absence of a dense matter background, collective neutrino oscillations are self-suppressed near the neutrino spheres where the neutrino density is large [8]. In the single-bulb model where all neutrino species have an identical angular emission, collective neutrino oscillations can take place but only in the parameter space where the matter potential is finely matched with the neutrino potential which is not the case in our example. In contrast, in the multibulb model which has a crossed neutrino angular distribution, fast flavor conversions can occur near the neutrino spheres for a broad range of matter densities spanning over two orders of magnitude. Since the actual matter profile falls into the regime of the flavor instability in this particular example, we expect that the fast flavor conversion can develop there.

\section{DISCUSSION AND CONCLUSIONS}

We have used the four-beam neutrino model to explain the origin of fast neutrino oscillations in a dense, anistropic neutrino gas even in the limit that the neutrino mass splitting $\delta m^{2} \rightarrow 0$, although a finite flavor mixing is necessary to initiate such a flavor conversion. It is the multiangle effect of the neutrinos as they travel through a dense matter and neutrino background that induce the fast flavor conversion. This flavor conversion can take place inside a very dense medium over very short distance scales of order $\left(G_{\mathrm{F}} n_{e}\right)^{-1}$ or $\left(G_{\mathrm{F}} n_{\nu}\right)^{-1}$.

We have shown that the fast flavor conversion is generally prohibited in a stationary, outward flowing, axially symmetric neutrino flux unless there is a crossing in the neutrino angular distribution. This provides an explicit proof for a similar conjecture by Dasgupta et al. in Ref. [14] for the multibulb supernova model. However, this conclusion does not apply to a medium where both inward and outward flowing neutrino fluxes are present. This can be easily seen from the four-beam model when the direction of two of the neutrino beams are flipped, e.g., $v_{2 z} \rightarrow-v_{2 z}$ and $v_{3 z} \rightarrow-v_{3 z}$. This would result a sign change in the effective spectrum

$$
\tilde{g}_{1 \pm}= \pm \frac{g_{1}}{v_{2 z}} \rightarrow-\tilde{g}_{1 \pm}
$$

[see Eqs. (26) and (29)]. Therefore, even if there is no crossing in the actual angular distribution $G\left(v_{z}\right)$, there can still be a crossing in the effective spectrum $\tilde{g}(\tilde{\omega})$ because of this sign change in the part of the effective spectrum for the inward neutrino fluxes. In other words, fast flavor conversions can exist in a stationary neutrino gas with both inward and outward neutrino fluxes, e.g., inside the $\nu_{e}$ sphere of a core-collapse supernova, even if there is no crossing in the neutrino angular distribution.

We note that the convective operator in the EoM (5) becomes

$$
\partial_{t}+\mathbf{v} \cdot \boldsymbol{\nabla} \rightarrow \mathrm{i}(-\Omega+\mathbf{v} \cdot \mathbf{K})
$$

for a collective oscillation wave of collective frequency $\Omega$ and wave vector $\mathbf{K}[15,27]$. It is the different signs in $\mathbf{v} \cdot \mathbf{K}$ of the inward and outward neutrino fluxes that produce the crossing in the effective spectrum $\tilde{g}(\tilde{\omega})$ in a stationary neutrino gas when there is no crossing in the actual neutrino angular distribution. Obviously, this argument does not apply to the temporal flavor instability (i.e., with $\operatorname{Im}(\Omega) \neq 0$ ) which still requires a crossed neutrino angular distribution [15].

We have applied the linear flavor stability analysis to a multibulb supernova model. As envisioned by Sawyer fast flavor conversions can take place in this model because different neutrino species have different neutrino spheres. However, it is somewhat surprising that it is because of the presence of a large matter density that this flavor conversion is possible. It has been pointed out that the matter effect can be (partially) "cancelled" in a nonstationary neutrino gas $[28,29]$. However, a significant cancellation of the matter effect occurs only for rapidly pulsating neutrino oscillation modes of frequency $\sim \lambda$ which is extremely high near or inside the neutrino sphere. The matter effect discussed in this work is still relevant to most other neutrino 
oscillation modes which are probably more important in the real supernova environment.

Of course, our result about supernova neutrinos is only suggestive because of the various assumptions of the multibulb model. A real supernova is dynamic and does not have clear-cut neutrino spheres. There also exist neutrinos propagating inward which can affect neutrino flavor conversions [15,30]. A more definite conclusion requires simulations of neutrino flavor oscillations in timedependent, multidimensional supernova models with accurate neutrino angular distributions.

\section{ACKNOWLEDGMENTS}

We thank L. Ma, J. Martin, S. Shalgar, and Y.-Z. Qian for useful discussions and G. Raffelt for reading the manuscript and providing valuable suggestions. We also thank T. Janka for providing various supernova models. H. D. would like to thank the hospitality of YITP, Kyoto where this work was started and the nuclear physics group at UMN where it was finished. This work was supported by DOE EPSCoR Grant No. DE-SC0008142 and DOE NP Grant No. DESC0017803 at UNM.

\section{APPENDIX: AXIALLY SYMMETRIC FAST OSCILLATIONS IN AN OUTWARD FLOWING NEUTRINO FLUX}

To find out the criterion for the occurrence of the axially symmetric fast flavor conversions in an outward flowing neutrino flux (Sec. IV B), we again assume that there exists a flavor instability for $\mu$ within $\left(\mu_{-}, \mu_{+}\right)$. As $\mu \rightarrow \mu_{-}+0^{+}$, $\Omega \rightarrow \gamma_{\mathrm{c}}$, and

$$
I\left[v_{z}^{m}\right] \rightarrow \frac{\mu}{2}\left[P\left[v_{z}^{m}\right]+\mathrm{i} \pi G\left(v_{\mathrm{c}}\right) \frac{v_{\mathrm{c}}^{m+1}}{\bar{\lambda}}\right],
$$

where

$$
v_{\mathrm{c}}=\frac{\bar{\lambda}}{\Phi_{z}-\gamma_{\mathrm{c}}},
$$

$$
P\left[f\left(v_{z}\right)\right]=\mathcal{P} \int_{0}^{1} \frac{G\left(v_{z}\right) f\left(v_{z}\right) \mathrm{d} v_{z}}{\left(\gamma_{\mathrm{c}}-\Phi_{z}\right) v_{z}+\bar{\lambda}}
$$

At this limit, the imaginary part of Eq. (39a) can be written as

$$
\left(P\left[\left(v_{z}-v_{\mathrm{c}}\right)^{2}\right]+\frac{1-v_{\mathrm{c}}^{2}}{\mu_{-} / 2}\right) G\left(v_{\mathrm{c}}\right) v_{\mathrm{c}}=0
$$

which is possible if $G\left(v_{\mathrm{c}}\right)=0$.

If $G\left(v_{z}\right)$ has only one simple crossing point (at $v_{\mathrm{c}}$ ) within the range of $[0,1]$, then function

$$
\frac{G\left(v_{z}\right)}{\left(\gamma_{\mathrm{c}}-\Phi_{z}\right) v_{z}+\bar{\lambda}}=\frac{1}{\gamma_{\mathrm{c}}-\Phi_{z}} \frac{G\left(v_{z}\right)}{v_{z}-v_{\mathrm{c}}}
$$

has no crossing within the same range. In this case, we can define

$$
\left\langle f\left(v_{z}\right)\right\rangle=\frac{P\left[f\left(v_{z}\right)\right]}{P[1]}
$$

as an average of $f\left(v_{z}\right)$ within $[0,1]$, and the real part of Eq. (39a) implies that

$\frac{4 / \mu_{-}}{P[1]}=\left(1-\left\langle v_{z}^{2}\right\rangle\right) \pm \sqrt{\left(1-\left\langle v_{z}^{2}\right\rangle\right)^{2}+4\left(\left\langle v_{z}^{2}\right\rangle-\left\langle v_{z}\right\rangle^{2}\right)}$.

The two solutions in the above equation require opposite crossing directions of $G\left(v_{z}\right)$ at $v_{\mathrm{c}}$.

Therefore, a condition for the axially symmetric flavor oscillations to occur in an outward flowing neutrino flux is that its angular distribution $G\left(v_{z}\right)$ has a crossing point within the range of $[0,1]$, but the sign of this crossing is not constrained.
[1] C. Patrignani et al. (Particle Data Group), Chin. Phys. C 40, 100001 (2016).

[2] S. P. Mikheyev and A. Y. Smirnov, Yad. Fiz. 42, 1441 (1985) [Sov. J. Nucl. Phys. 42, 913 (1985)].

[3] R. F. Sawyer, Phys. Rev. D 72, 045003 (2005).

[4] R. F. Sawyer, Phys. Rev. D 79, 105003 (2009).

[5] H. Duan, G. M. Fuller, J. Carlson, and Y.-Z. Qian, Phys. Rev. D 74, 105014 (2006).

[6] A. Esteban-Pretel, S. Pastor, R. Tomas, G. G. Raffelt, and G. Sigl, Phys. Rev. D 76, 125018 (2007).
[7] G. L. Fogli, E. Lisi, A. Marrone, and A. Mirizzi, J. Cosmol. Astropart. Phys. 12 (2007) 010.

[8] H. Duan and A. Friedland, Phys. Rev. Lett. 106, 091101 (2011).

[9] A. Mirizzi and P. D. Serpico, Phys. Rev. Lett. 108, 231102 (2012).

[10] A. Mirizzi and P. D. Serpico, Phys. Rev. D 86, 085010 (2012).

[11] H. Duan, G. M. Fuller, and Y.-Z. Qian, Annu. Rev. Nucl. Part. Sci. 60, 569 (2010).

[12] R. F. Sawyer, Phys. Rev. Lett. 116, 081101 (2016). 
[13] S. Chakraborty, R. S. Hansen, I. Izaguirre, and G. Raffelt, J. Cosmol. Astropart. Phys. 03 (2016) 042.

[14] B. Dasgupta, A. Mirizzi, and M. Sen, J. Cosmol. Astropart. Phys. 02 (2017) 019.

[15] I. Izaguirre, G. Raffelt, and I. Tamborra, Phys. Rev. Lett. 118, 021101 (2017).

[16] F. Capozzi, B. Dasgupta, E. Lisi, A. Marrone, and A. Mirizzi, Phys. Rev. D 96, 043016 (2017).

[17] B. Dasgupta and M. Sen, Phys. Rev. D 97, 023017 (2018).

[18] H. Duan, G. M. Fuller, and Y.-Z. Qian, Phys. Rev. D 74, 123004 (2006).

[19] B. Dasgupta, A. Dighe, G. G. Raffelt, and A. Y. Smirnov, Phys. Rev. Lett. 103, 051105 (2009).

[20] A. Banerjee, A. Dighe, and G. Raffelt, Phys. Rev. D 84, 053013 (2011).

[21] G. Sigl and G. Raffelt, Nucl. Phys. B406, 423 (1993).
[22] V. A. Kostelecký and S. Samuel, Phys. Rev. D 52, 621 (1995).

[23] S. Hannestad, G. G. Raffelt, G. Sigl, and Y. Y. Y. Wong, Phys. Rev. D 74, 105010 (2006).

[24] H. Duan, G. M. Fuller, J. Carlson, and Y.-Z. Qian, Phys. Rev. D 75, 125005 (2007).

[25] A. Esteban-Pretel, A. Mirizzi, S. Pastor, R. Tomàs, G. G. Raffelt, P. D. Serpico, and G. Sigl, Phys. Rev. D 78, 085012 (2008).

[26] L. Hudepohl, B. Muller, H.-T. Janka, A. Marek, and G. Raffelt, Phys. Rev. Lett. 104, 251101 (2010).

[27] H. Duan, G. M. Fuller, and Y.-Z. Qian, arXiv:0808.2046v1.

[28] S. Abbar and H. Duan, Phys. Lett. B 751, 43 (2015).

[29] B. Dasgupta and A. Mirizzi, Phys. Rev. D 92, 125030 (2015).

[30] J. F. Cherry, J. Carlson, A. Friedland, G. M. Fuller, and A. Vlasenko, Phys. Rev. Lett. 108, 261104 (2012). 